\title{
THE COMPLETE DIRICHLET-TO-NEUMANN MAP FOR DIFFERENTIAL FORMS
}

\author{
VLADIMIR SHARAFUTDINOV AND CLAYTON SHONKWILER
}

\begin{abstract}
The Dirichlet-to-Neumann map for differential forms on a Riemannian manifold with boundary is a generalization of the classical Dirichlet-to-Neumann map which arises in the problem of Electrical Impedance Tomography. We synthesize the two different approaches to defining this operator by giving an invariant definition of the complete Dirichlet-to-Neumann map for differential forms in terms of two linear operators $\Phi$ and $\Psi$. The pair $(\Phi, \Psi)$ is equivalent to Joshi and Lionheart's operator $\Pi$ and determines Belishev and Sharafutdinov's operator $\Lambda$. We show that the Betti numbers of the manifold are determined by $\Phi$ and that $\Psi$ determines a chain complex whose homologies are explicitly related to the cohomology groups of the manifold.
\end{abstract}

\section{INTRODUCTION}

We consider the problem of recovering the topology of a compact, oriented, smooth Riemannian manifold $(M, g)$ with boundary from the Dirichletto-Neumann map for differential forms. The classical Dirichlet-to-Neumann map for functions was first defined by Calderón [Cal80], and has been shown to recover surfaces up to conformal equivalence [LU01, Bel03] and realanalytic manifolds of dimension $\geq 3$ up to isometry [LTU03].

The classical Dirichlet-to-Neumann map was generalized to an operator on differential forms independently by Joshi and Lionheart [JL05] and Belishev and Sharafutdinov [BS08]. Joshi and Lionheart called their operator $\Pi$ and showed that the data $(\partial M, \Pi)$ determines the $C^{\infty}$-jet of the Riemannian metric at the boundary. Krupchyk, Lassas, and Uhlmann have recently extended this result to show that $(\partial M, \Pi)$ determines a real-analytic manifold up to isometry [KLU10].

On the other hand, Belishev and Sharafutdinov called their Dirichletto-Neumann map $\Lambda$ and showed that $(\partial M, \Lambda)$ determines the cohomology groups of the manifold $M$. Shonkwiler [Sho09] demonstrated a connection between $\Lambda$ and invariants called Poincaré duality angles and showed that the cup product structure of the manifold $M$ can be partially recovered from $(\partial M, \Lambda)$.

Date: October 12, 2018.

2000 Mathematics Subject Classification. Primary: 58A14, 58J32; Secondary: 57R19.

Key words and phrases. Hodge theory, inverse problems, Dirichlet-to-Neumann map. 
The operators $\Pi$ and $\Lambda$ are similar, but do not appear to be equivalent. One of the advantages of Belishev and Sharafutdinov's $\Lambda$, especially for the task of recovering topological data, is that it is defined invariantly. In this paper we provide an invariant definition of Joshi and Lionheart's operator $\Pi$, which we give in terms of two auxiliary operators

$$
\Phi: \Omega^{k}(\partial M) \rightarrow \Omega^{n-k-1}(\partial M) \text { and } \Psi: \Omega^{k}(\partial M) \rightarrow \Omega^{k-1}(\partial M) .
$$

We can easily show that $\Lambda$ is determined by $\Phi$ and $\Psi$, so it makes sense to regard $\Pi$ as the "complete" Dirichlet-to-Neumann operator on differential forms.

Belishev and Sharafutdinov's proof that the Betti numbers of $M$ can be recovered from the data $(\partial M, \Lambda)$ was somewhat circuitous, as it involved determining the dimension of the image of the operator $G=\Lambda \pm d_{\partial} \Lambda^{-1} d_{\partial}$. In contrast, it is straightforward to recover the Betti numbers of $M$ from $\Phi$.

Theorem 1. Let $\beta_{k}(M)=\operatorname{dim} H^{k}(M ; \mathbb{R})$ be the kth Betti number of $M$. Then

$$
\beta_{k}(M)=\operatorname{dim} \operatorname{ker} \Phi
$$

The operator $\Psi$ turns out to be a chain map and the homology of the chain complex $\left(\Omega^{*}(\partial M), \Psi\right)$ is given in terms of a mixture of absolute and relative cohomology groups of $M$.

Theorem 2. For any $0 \leq k \leq n-1$,

$$
H_{k}\left(\Omega^{*}(\partial M), \Psi\right) \simeq H^{k+1}(M, \partial M ; \mathbb{R}) \oplus H^{k}(M ; \mathbb{R}) .
$$

This, in turn, implies that the space of $k$-forms on $\partial M$ contains an "echo" (detected by $\Pi$ ) of the $(k+1)$ st relative cohomology group of $M$.

Corollary 3. The space $\Omega^{k}(\partial M)$ of $k$-forms on $\partial M$ contains a subspace isomorphic to $H^{k+1}(M, \partial M ; \mathbb{R})$ which is distinguished by the Dirichlet-toNeumann operator $\Pi$. Specifically,

$$
\left(\operatorname{ker} \Psi_{k} / \operatorname{im} \Psi_{k+1}\right) / \operatorname{ker} \Phi_{k} \simeq H^{k+1}(M, \partial M ; \mathbb{R}) .
$$

When $n=2$ and $k=0$, Theorem 1 and Corollary 3 imply that all the cohomology groups of a surface are contained in $\Omega^{0}(\partial M)$.

Corollary 4. All of the cohomology groups of a surface $M$ with boundary can be realized inside the space of smooth functions on $\partial M$, where they can be recovered by the Dirichlet-to-Neumann operator $\Pi$.

Since $\Psi$ is a chain map, it is natural to try to define associated cochain maps and compute their cohomologies. In this spirit, we define $\widetilde{\Psi}= \pm \star_{\partial} \Psi \star_{\partial}$ and show that it is the adjoint of $\Psi$. Not surprisingly,

$$
H^{k}\left(\Omega^{*}(\partial M), \widetilde{\Psi}\right) \simeq H_{n-k-1}\left(\Omega^{*}(\partial M), \Psi\right) .
$$

Finally, we define another cochain map $\Theta$ with the same cohomology as $\widetilde{\Psi}$. It turns out that $\Theta= \pm d_{\partial} \Phi^{2}$, so the cohomology of $\widetilde{\Psi}$ (and hence the homology of $\Psi$ ) is completely determined by the operator $\Phi$. With this in 
mind, restating Corollary 3 in terms of $\Phi$ and specializing to the case $k=0$ yields the following:

Corollary 5. A copy of the cohomology group $H^{n-1}(M ; \mathbb{R})$ is distinguished by the operator $\Phi$ inside $\Omega^{0}(\partial M)$, the space of smooth functions on $\partial M$. Specifically,

$$
\operatorname{ker}\left(d_{\partial} \Phi^{2}\right) / \operatorname{ker} \Phi \simeq H^{n-1}(M ; \mathbb{R})
$$

The above results all suggest that the operator $\Pi$ (and, in particular, $\Phi$ ) encodes more information about the topology of $M$ than does the operator $\Lambda$. Thus far nobody has been able to use $\Lambda$ to recover the cohomology ring structure on $M$, but perhaps this will be easier to recover from the operator П. Another interesting question relates to the linearized inverse problem of recovering the metric: can the results of [Sha09] be strengthened if the data $\Lambda$ are replaced with the richer data $(\Phi, \Psi)$ ?

\section{The operators $\Phi$ AND $\Psi$}

Throughout this paper, $(M, g)$ will be a smooth, compact, oriented Riemannian manifold of dimension $n \geq 2$ with nonempty boundary. The term "smooth" is used as a synonym for " $C^{\infty}$-smooth". Let $i: \partial M \hookrightarrow M$ be the identical embedding and let $\Omega(M)=\bigoplus_{k=0}^{n} \Omega^{k}(M)$ be the graded algebra of smooth differential forms on $M$. We use the standard operators $d, \delta, \Delta$, and $\star$ on $\Omega(M)$, as well as their analogues $d_{\partial}, \delta_{\partial}, \Delta_{\partial}$, and $\star_{\partial}$ on $\Omega(\partial M)$.

Joshi and Lionheart defined their Dirichlet-to-Neumann operator

$$
\Pi:\left.\left.\Omega(M)\right|_{\partial M} \rightarrow \Omega(M)\right|_{\partial M}
$$

as

$$
\Pi \chi:=\left.\frac{\partial \omega}{\partial \nu}\right|_{\partial M},
$$

where $\nu$ is the unit outward normal vector at the boundary and $\omega$ is the solution to the boundary value problem

$$
\left\{\begin{array}{l}
\Delta \omega=0 \\
\left.\omega\right|_{\partial M}=\chi .
\end{array}\right.
$$

This boundary value problem has a unique solution for every $\left.\chi \in \Omega(M)\right|_{\partial M}$ [Sch95, Theorem 3.4.1].

When applied to forms, the meaning of the normal derivative $\partial / \partial \nu$ needs to be specified. Instead, we prefer to give an equivalent definition of $\Pi$ in invariant terms. To do so, note that the restriction $\left.\omega\right|_{\partial M}$ is determined by two boundary forms, $i^{*} \omega$ and $i^{*} \star \omega$. Likewise, the data $\partial \omega /\left.\partial \nu\right|_{\partial M}$ are equivalent to the two boundary forms $i^{*} \star d \omega$ and $i^{*} \delta \omega$. Hence, we will define the operator

$$
\Pi: \Omega^{k}(\partial M) \times \Omega^{n-k}(\partial M) \rightarrow \Omega^{n-k-1}(\partial M) \times \Omega^{k-1}(\partial M)
$$


by

$$
\Pi\left(\begin{array}{l}
\varphi \\
\psi
\end{array}\right)=\left(\begin{array}{c}
i^{*} \star d \omega \\
i^{*} \delta \omega
\end{array}\right)
$$

where $\omega \in \Omega^{k}(M)$ is the solution to the boundary value problem

$$
\left\{\begin{array}{l}
\Delta \omega=0 \\
i^{*} \omega=\varphi, \quad i^{*} \star \omega=\psi .
\end{array}\right.
$$

Since $\Pi$ sends pairs of forms to pairs of forms, it is somewhat cumbersome to work with in practice. Instead of using it directly, we find a pair of operators $(\Phi, \Psi)$ which is equivalent to $\Pi$. Define the linear operators

$$
\Phi: \Omega^{k}(\partial M) \rightarrow \Omega^{n-k-1}(\partial M) \text { and } \Psi: \Omega^{k}(\partial M) \rightarrow \Omega^{k-1}(\partial M)
$$

by the equalities

$$
\Phi \varphi=i^{*} \star d \omega \text { and } \Psi \varphi=i^{*} \delta \omega .
$$

Here $\omega \in \Omega^{k}(M)$ is the solution to the boundary value problem

$$
\left\{\begin{array}{l}
\Delta \omega=0 \\
i^{*} \omega=\varphi, \quad i^{*} \star \omega=0 .
\end{array}\right.
$$

Now it is straightforward to express $\Pi$ in terms of $\Phi$ and $\Psi$. We write $\Pi$ as the matrix

$$
\Pi=\left(\begin{array}{ll}
\Pi_{11} & \Pi_{12} \\
\Pi_{21} & \Pi_{22}
\end{array}\right) .
$$

Then, comparing (1) and (3),

$$
\Pi_{11}=\Phi, \quad \Pi_{21}=\Psi .
$$

From (1) and (2), the operators $\Pi_{12}$ and $\Pi_{22}$ are given by

$$
\Pi_{12} \psi=i^{*} \star d \varepsilon \quad \text { and } \Pi_{22} \psi=i^{*} \delta \varepsilon
$$

where $\varepsilon$ solves the boundary value problem

$$
\left\{\begin{array}{l}
\Delta \varepsilon=0 \\
i^{*} \varepsilon=0, \quad i^{*} \star \varepsilon=\psi .
\end{array}\right.
$$

If $\varepsilon \in \Omega^{k}(M)$ is the solution to this boundary value problem for $\psi \in$ $\Omega^{n-k}(\partial M)$, then the form $\omega=\star \varepsilon$ solves the problem

$$
\left\{\begin{array}{l}
\Delta \omega=0 \\
i^{*} \omega=\psi, \quad i^{*} \star \omega=0 .
\end{array}\right.
$$

Comparing this to (4), we see that

$$
\Phi \psi=i^{*} \star d \omega \text { and } \Psi \psi=i^{*} \delta \omega .
$$

Since

$$
i^{*} \star d \omega=(-1)^{n(n-k)+1} i^{*} \delta \varepsilon \quad \text { and } \quad i^{*} \delta \omega=(-1)^{k+1} i^{*} \star d \varepsilon,
$$


(1) and (5) imply that

$$
\Pi_{12}=(-1)^{n(n-k)+1} \Psi \quad \text { and } \quad \Pi_{22}=(-1)^{k+1} \Phi \quad \text { on } \quad \Omega^{n-k}(\partial M) .
$$

Therefore, the operator $\Pi$ can be expressed in terms of $\Phi$ and $\Psi$ as

$$
\Pi=\left(\begin{array}{cc}
\Phi & (-1)^{n(n-k)+1} \Psi \\
\Psi & (-1)^{k+1} \Phi
\end{array}\right) \quad \text { on } \quad \Omega^{k}(\partial M) \times \Omega^{n-k}(\partial M) .
$$

Belishev and Sharafutdinov's version of the Dirichlet-to-Neumann map is the operator

$$
\Lambda: \Omega^{k}(\partial M) \rightarrow \Omega^{n-k-1}(\partial M)
$$

given by

$$
\Lambda \varphi=i^{*} \star d \omega,
$$

where $\omega \in \Omega^{k}(M)$ is a solution to the boundary value problem

$$
\left\{\begin{array}{l}
\Delta \omega=0 \\
i^{*} \omega=\varphi, \quad i^{*} \delta \omega=0 .
\end{array}\right.
$$

We can now express the operator $\Lambda$ in terms of $\Phi$ and $\Psi$. Given $\varphi \in$ $\Omega^{k}(\partial M)$, let $\omega \in \Omega^{k}(M)$ solve the boundary value problem (7) and set $\psi=i^{*} \star \omega$. Then $\omega$ solves the boundary value problem (2), so we have that

$$
\Pi\left(\begin{array}{c}
\varphi \\
\psi
\end{array}\right)=\left(\begin{array}{c}
i^{*} \star d \omega \\
i^{*} \delta \omega
\end{array}\right)=\left(\begin{array}{c}
\Lambda \varphi \\
0
\end{array}\right)
$$

With the help of (6) we can rewrite this equation as the system

$$
\begin{aligned}
\Phi \varphi+(-1)^{n(n-k)+1} \Psi \psi & =\Lambda \varphi \\
\Psi \varphi+(-1)^{k+1} \Phi \psi & =0 .
\end{aligned}
$$

Eliminating $\psi$ from the system yields the expression

$$
\Lambda=\Phi+(-1)^{n(n-k)+k+1} \Psi \Phi^{-1} \Psi \quad \text { on } \quad \Omega^{k}(\partial M) .
$$

The fact that the operator $\Psi \Phi^{-1} \Psi$ is well-defined follows from Corollary 4.3, stated below.

We take this opportunity to record some useful relations involving $\Phi$ and $\Psi$ :

Lemma 2.1. The operators $\Phi$ and $\Psi$ satisfy the following relations:

$$
\begin{aligned}
\Phi \Psi & =(-1)^{k} d_{\partial} \Phi \quad \text { on } \quad \Omega^{k}(\partial M), \\
\Psi^{2} & =0 \\
\Psi \Phi & =(-1)^{k+1} \Phi d_{\partial} \quad \text { on } \quad \Omega^{k}(\partial M), \\
\Phi^{2} & =(-1)^{k n}\left(d_{\partial} \Psi+\Psi d_{\partial}\right) \quad \text { on } \quad \Omega^{k}(\partial M)
\end{aligned}
$$


Proof. Given $\varphi \in \Omega^{k}(\partial M)$, let $\omega \in \Omega^{k}(M)$ solve the boundary value problem (4). Then

$$
\Phi \varphi=i^{*} \star \omega, \quad \Psi \varphi=i^{*} \delta \omega .
$$

Letting $\xi=\delta \omega$, we certainly have $\Delta \xi=0$. Pulling $\xi$ and $\star \xi$ back to the boundary yields

$$
\begin{aligned}
i^{*} \xi & =i^{*} \delta \omega=\Psi \varphi \\
i^{*} \star \xi & =i^{*} \star \delta \omega= \pm i^{*} d \star \omega= \pm d_{\partial} i^{*} \star \omega=0 .
\end{aligned}
$$

Therefore, $\xi$ solves the boundary value problem

$$
\left\{\begin{array}{l}
\Delta \xi=0 \\
i^{*} \xi=\Psi \varphi, \quad i^{*} \star \xi=0,
\end{array}\right.
$$

and so

$$
\Phi \Psi \varphi=i^{*} \star d \xi \quad \text { and } \quad \Psi^{2} \varphi=i^{*} \delta \xi .
$$

Since $\Delta \omega=0$, it follows that $d \delta \omega=-\delta d \omega$, which we use to see that

$$
\begin{aligned}
i^{*} \star d \xi & =i^{*} \star d \delta \omega=-i^{*} \star \delta d \omega=(-1)^{k} i^{*} d \star d \omega=(-1)^{k} d_{\partial} i^{*} \star d \omega, \\
i^{*} \delta \xi & =i^{*} \delta \delta \omega=0 .
\end{aligned}
$$

Comparing this with (14), we obtain

$$
\Phi \Psi \varphi=(-1)^{k} d_{\partial} i^{*} \star d \omega \text { and } \Psi^{2} \varphi=0 .
$$

With the help of (13), this gives (9) and (10).

Turning to (11), we again let $\omega \in \Omega^{k}(M)$ solve (4) for a form $\varphi \in \Omega^{k}(\partial M)$. Let $\varepsilon \in \Omega^{k+1}(M)$ be a solution to the problem

$$
\left\{\begin{array}{l}
\Delta \varepsilon=0 \\
i^{*} \varepsilon=d \varphi, \quad i^{*} \star \varepsilon=0 .
\end{array}\right.
$$

Then

$$
\Phi d_{\partial} \varphi=i^{*} \star d \varepsilon, \quad \Psi d_{\partial} \varphi=i^{*} \delta \varepsilon .
$$

Define $\eta \in \Omega^{n-k-1}(M)$ by

$$
\eta=\star d \omega-\star \varepsilon
$$

Clearly, $\Delta \eta=0$. Moreover,

$$
\star \eta=\star \star(d \omega-\varepsilon)= \pm(d \omega-\varepsilon),
$$

so

$$
i^{*} \star \eta= \pm i^{*}(d \omega-\varepsilon)= \pm(d \varphi-d \varphi)=0 .
$$

Also,

$$
i^{*} \eta=i^{*} \star d \omega-i^{*} \star \varepsilon=\Phi \varphi,
$$

since $i^{*} \star \varepsilon=0$. 
Therefore, $\eta$ solves the boundary value problem

$$
\left\{\begin{array}{l}
\Delta \eta=0 \\
i^{*} \eta=\Psi \varphi, \quad i^{*} \star \eta=0 .
\end{array}\right.
$$

Hence,

$$
\Phi^{2} \varphi=i^{*} \star d \eta \text { and } \Phi \Psi \varphi=i^{*} \delta \eta .
$$

Using (16) we see that

$$
\delta \eta=\delta \star d \omega-\delta \star \varepsilon= \pm \star d d \omega-\delta \star \varepsilon=(-1)^{k+1} \star d \varepsilon .
$$

Thus,

$$
i^{*} \delta \eta=(-1)^{k+1} i^{*} \star d \epsilon,
$$

which, along with (15) and (17), yields

$$
\Psi \Phi \varphi=(-1)^{k+1} \Phi d_{\partial} \varphi
$$

proving (11).

Finally, (12) is proved along the same lines. From (16) we have

$$
\star d \eta=\star d \star(d \omega-\varepsilon)=(-1)^{k n+1}(\delta d \omega-\delta \varepsilon) .
$$

Again making use of the fact that $\delta d \omega=-d \delta \omega$, this implies that

$$
i^{*} \star d \omega=(-1)^{k n+1}\left(i^{*} \delta d \omega-i^{*} \delta \varepsilon\right)=(-1)^{k n}\left(d_{\partial} i^{*} \delta \omega+i^{*} \delta \varepsilon\right) .
$$

In turn, we can use (13) and (15) to rewrite the above formula as

$$
i^{*} \star d \eta=(-1)^{k n}\left(d_{\partial} \Psi \varphi+\Psi d_{\partial} \varphi\right) .
$$

Comparing with (17), this produces the desired relation (12).

Remark 2.2. The key properties of the operator $\Lambda$ are expressed by the equalities

$$
\Lambda d_{\partial}=0, \quad d_{\partial} \Lambda=0, \quad \text { and } \quad \Lambda^{2}=0 .
$$

It is straightforward to check that these equalities follow from (8) and Lemma 2.1.

\section{Recovering the Betti numbers of $M$ From $\Phi$}

Belishev and Sharafutdinov showed that the Betti numbers of the manifold $M$,

$$
\beta_{k}(M)=\operatorname{dim} H^{k}(M ; \mathbb{R}),
$$

can be recovered from the data $(\partial M, \Lambda)$. The proof of this fact is somewhat indirect, involving the auxiliary operator

$$
G=\Lambda+(-1)^{k n+k+n} d_{\partial} \Lambda^{-1} d_{\partial}: \Omega^{k}(\partial M) \rightarrow \Omega^{n-k-1}(\partial M) .
$$

In contrast, it is much more straightforward to recover the Betti numbers of $M$ from the operator $\Phi$.

Theorem 1. Let $\Phi_{k}: \Omega^{k}(\partial M) \rightarrow \Omega^{n-k-1}(\partial M)$ be the restriction of $\Phi$ to $\Omega^{k}(\partial M)$. Then

$$
\beta_{k}(M)=\operatorname{dim} \operatorname{ker} \Phi_{k}
$$


The Hodge-Morrey-Friedrichs decomposition theorem [Sch95, Section 2.4] implies that

$$
H^{k}(M ; \mathbb{R}) \simeq \mathcal{H}_{N}^{k}(M)
$$

where

$$
\mathcal{H}_{N}^{k}(M):=\left\{\omega \in \Omega^{k}(M): d \omega=0, \delta \omega=0, i^{*} \star \omega=0\right\}
$$

is the space of harmonic Neumann fields. Since harmonic forms are uniquely determined by their boundary values, $\mathcal{H}_{N}^{k}(M) \simeq i^{*} \mathcal{H}_{N}^{k}(M)$, so Theorem 1 is an immediate consequence of the following lemma.

Lemma 3.1. The kernel of the operator $\Phi_{k}: \Omega^{k}(\partial M) \rightarrow \Omega^{n-k-1}(\partial M)$ consists of the boundary traces of harmonic Neumann fields; i.e.,

$$
\operatorname{ker} \Phi_{k}=i^{*} \mathcal{H}_{N}^{k}(M) \text {. }
$$

The image of $\Phi_{k}$ coincides with the subspace $\left(i^{*} \mathcal{H}_{N}^{k}(M)\right)^{\perp} \subset \Omega^{n-k-1}(\partial M)$ consisting of forms $\psi \in \Omega^{n-k-1}(\partial M)$ satisfying

$$
\int_{\partial M} \psi \wedge \chi=0 \quad \forall \xi \in i^{*} \mathcal{H}_{N}^{k}(M)
$$

In particular, $\Phi$ is a Fredholm operator with index zero.

Proof. If $\varphi \in \Omega^{k}(\partial M)$ such that $\Phi_{k} \varphi=0$, then the boundary value problem

$$
\left\{\begin{array}{l}
\Delta \omega=0 \\
i^{*} \omega=\varphi, \quad i^{*} \star \omega=0, \quad i^{*} \star d \omega=0
\end{array}\right.
$$

is solvable. Using Green's formula,

$$
\langle d \omega, d \omega\rangle_{L^{2}}+\langle\delta \omega, \delta \omega\rangle_{L^{2}}=\langle\Delta \omega, \omega\rangle_{L^{2}}+\int_{\partial M} i^{*}(\omega \wedge \star d \omega-\delta \omega \wedge \star \omega) .
$$

The right side of this equation equals zero since $\omega$ solves the boundary value problem (20). Hence, $\omega$ is a harmonic Neumann field since $i^{*} \star \omega=0$, and so $\varphi=i^{*} \omega \in i^{*} \mathcal{H}_{N}^{k}(M)$.

The converse statement is immediate: if $\varphi=i^{*} \omega$ for $\omega \in \mathcal{H}_{N}^{k}(M)$, then $\omega$ solves the boundary value problem (20) and hence $\varphi \in \operatorname{ker} \Phi_{k}$.

On the other hand, a form $\psi \in \Omega^{n-k-1}(\partial M)$ is in the image of $\Phi_{k}$ if and only if the boundary value problem

$$
\left\{\begin{array}{l}
\Delta \omega=0 \\
i^{*} \star \omega=0, \quad i^{*} \star d \omega=\psi
\end{array}\right.
$$

is solvable. The defining condition (19) of $\left(i^{*} \mathcal{H}_{N}^{k}(M)\right)^{\perp}$ is precisely the necessary and sufficient condition for the solvability of this boundary value problem [Sch95, Corollary 3.4.8].

Corollary 3.2. The operator $d_{\partial} \Phi^{-1}$ is well-defined on $\operatorname{im} \Phi_{k}=\left(i^{*} \mathcal{H}_{N}^{k}(M)\right)^{\perp}$; i.e., the equation $\Phi \varphi=\psi$ has a solution $\varphi$ for every $\psi \in\left(i^{*} \mathcal{H}_{N}^{k}(M)\right)^{\perp}$ and $d_{\partial} \varphi$ is uniquely determined by $\psi$. 
Proof. A form $\psi \in\left(i^{*} \mathcal{H}_{N}^{k}(M)\right)^{\perp}$ belongs to the range of $\Phi$, so the equation $\Phi \varphi=\psi$ is solvable. If $\Phi \varphi_{1}=\Phi \varphi_{2}$, then the form $\varphi_{1}-\varphi_{2} \in \operatorname{ker} \Phi$ is closed, meaning that $d_{\partial} \varphi_{1}=d_{\partial} \varphi_{2}$.

The apparent similarity between the operator $d_{\partial} \Phi^{-1}$ and the Hilbert transform $T=d_{\partial} \Lambda^{-1}$ defined by Belishev and Sharafutdinov is no accident, as the following proposition demonstrates. Thus, the connection to the Poincaré duality angles of $M$ [Sho09, Theorem 4] comes directly from the definition of $\Phi$ (and hence $\Pi$ ) without using $\Lambda$ as an intermediary.

Proposition 3.3. $d_{\partial} \Lambda^{-1}=d_{\partial} \Phi^{-1}$, where the term on the right-hand side is understood to be the restriction of $d_{\partial} \Phi^{-1}$ to $\operatorname{im} \Lambda=i^{*} \mathcal{H}^{k}(M)$.

Proof. Suppose $\varphi \in \operatorname{im} \Lambda=i^{*} \mathcal{H}^{k}(M)$. Then $\varphi=i^{*} \omega$ for some $\omega \in \mathcal{H}^{k}(M)$. The Friedrichs decomposition says that

$$
\mathcal{H}^{k}(M)=c \mathcal{E} \mathcal{H}^{k}(M) \oplus \mathcal{H}_{D}^{k}(M),
$$

where

$$
\begin{aligned}
c \mathcal{E} \mathcal{H}^{k}(M) & =\left\{\delta \xi \in \Omega^{k}(M): d \delta \xi=0\right\} \\
\mathcal{H}_{D}^{k}(M) & =\left\{\eta \in \Omega^{k}(M): d \eta=0, \delta \eta=0, i^{*} \eta=0\right\} .
\end{aligned}
$$

Hence,

$$
\omega=\delta \xi+\eta \in c \mathcal{E} \mathcal{H}^{k}(M) \oplus \mathcal{H}_{D}^{k}(M) .
$$

The form $\xi \in \Omega^{k+1}(M)$ can be chosen such that $\xi$ is closed, $\Delta \xi=0$, and $i^{*} \xi=0$ [Sch95, p. 87, Remark 2]. Therefore,

$$
\left\{\begin{array}{l}
\Delta \star \xi=0, \\
i^{*} \star(\star \xi)=0, \\
i^{*} \delta \star \xi= \pm i^{*} \star d \star \star \xi= \pm i^{*} \star d \xi=0 .
\end{array}\right.
$$

This implies that $\star \xi$ solves the boundary value problems associated to both $\Lambda$ and $\Phi$, so

$$
\Lambda i^{*} \star \xi=i^{*} \star d \star \xi=(-1)^{n k+1} i^{*} \delta \xi=(-1)^{n k+1} i^{*} \omega=(-1)^{n k+1} \varphi
$$

and

$$
\Phi i^{*} \star \xi=i^{*} \star d \star \xi=(-1)^{n k+1} i^{*} \delta \xi=(-1)^{n k+1} i^{*} \omega=(-1)^{n k+1} \varphi .
$$

Hence,

$$
d \Lambda^{-1} \varphi=(-1)^{n k+1} d i^{*} \star \xi=d \Phi^{-1} i^{*} \star \xi,
$$

so we conclude that, indeed, $d \Lambda^{-1}=d \Phi^{-1}$. 


\section{The homology of the Chain complex $\left(\Omega^{*}(\partial M), \Psi\right)$}

We saw in Lemma 2.1 that $\Psi^{2}=0$, so it is natural to ask: what is the homology of the chain complex $\left(\Omega^{*}(\partial M), \Psi\right)$ ?

Theorem 2. For any $0 \leq k \leq n-1$, if $\Psi_{k}: \Omega^{k}(\partial M) \rightarrow \Omega^{k-1}(\partial M)$ is the restriction of $\Psi$ to the space of $k$-forms on $\partial M$, then

$$
H_{k}\left(\Omega^{*}(\partial M), \Psi\right)=\frac{\operatorname{ker} \Psi_{k}}{\operatorname{im} \Psi_{k+1}} \simeq H^{k+1}(M, \partial M ; \mathbb{R}) \oplus H^{k}(M ; \mathbb{R}) .
$$

In other words, the homology groups of $\left(\Omega^{*}(\partial M), \Psi\right)$ contain the absolute cohomology groups of $M$ in the same dimension and echoes of the relative cohomology groups of $M$ in one higher dimension. This behavior is similar to that exhibited by the cohomology of harmonic forms studied by Cappell, DeTurck, Gluck, and Miller [CDGM06].

Since $H^{k}(M ; \mathbb{R}) \simeq \operatorname{ker} \Phi_{k}$ (by Theorem 1) and since it will turn out that im $\Psi_{k+1}$ completely misses ker $\Phi_{k}$, we can see the echo of the $(k+1)$ st relative cohomology group of $M$ inside the space of $k$-forms on $\partial M$.

Corollary 3. The space $\Omega^{k}(\partial M)$ of $k$-forms on $\partial M$ contains a space isomorphic to $H^{k+1}(M, \partial M ; \mathbb{R})$ which is distinguished by the Dirichlet-to-Neumann operator $\Pi$. Specifically,

$$
\left(\operatorname{ker} \Psi_{k} / \operatorname{im} \Psi_{k+1}\right) / \operatorname{ker} \Phi_{k} \simeq H^{k+1}(M, \partial M ; \mathbb{R}) .
$$

When $n=2$ and $k=0$, Theorem 1 and Corollary 3 imply that $H^{0}(M ; \mathbb{R})$ and $H^{1}(M, \partial M ; \mathbb{R})$ can be distinguished inside the space of functions on $\partial M$. Moreover, by Poincaré-Lefschetz duality, $H^{0}(M ; \mathbb{R}) \simeq H^{2}(M, \partial M ; \mathbb{R})$ and $H^{1}(M, \partial M ; \mathbb{R}) \simeq H^{1}(M ; \mathbb{R})$. Since $H^{0}(M, \partial M ; \mathbb{R})$ and $H^{2}(M ; \mathbb{R})$ are both trivial, we have the following corollary.

Corollary 4. All of the cohomology groups of a surface $M$ with boundary can be realized inside the space of smooth functions on $\partial M$, where they can be recovered by the Dirichlet-to-Neumann operator $\Pi$.

Theorem 2 will follow from Lemmas 4.1 and 4.2, which describe the kernel and image of $\Psi$.

Lemma 4.1. If $\Psi_{k}: \Omega^{k}(\partial M) \rightarrow \Omega^{k-1}(\partial M)$ is the restriction of $\Psi$ to the space of $k$-forms on $\partial M$, then ker $\Psi_{k}$ is a direct sum of three spaces:

(i) The pullbacks of harmonic Neumann fields

$$
i^{*} \mathcal{H}_{N}^{k}(M)=\operatorname{ker} \Phi_{k}
$$

(ii) The space

$$
\operatorname{ker} G_{k} \cap i^{*}\left(\left(\mathcal{C}^{k}(M)\right)^{\perp}\right)
$$

which consists of the pullbacks of $k$-forms with conjugates on $M$ which are perpendicular to the space of closed forms.

(iii) A space isomorphic to $H^{k+1}(M, \partial M ; \mathbb{R})$. 
The operator $G_{k}$ is the restriction to $\Omega^{k}(\partial M)$ of the operator $G$ defined in (18).

Lemma 4.2. The image of the operator $\Psi_{k+1}: \Omega^{k+1}(\partial M) \rightarrow \Omega^{k}(\partial M)$ is precisely the space

$$
\operatorname{ker} G_{k} \cap i^{*}\left(\left(\mathcal{C}^{k}(M)\right)^{\perp}\right) .
$$

Proof of Lemma 4.1. Suppose $\varphi \in \Omega^{k}(\partial M)$ such that $\Psi \varphi=0$. Then, if $\omega \in \Omega^{k}(M)$ solves the boundary value problem (4), we have that

$$
0=\Psi \varphi=i^{*} \delta \omega .
$$

Using the Hodge-Morrey decomposition of $\Omega^{k}(M)$ [Sch95, Theorem 2.4.2],

$$
\omega=\delta \xi+\kappa+d \zeta \in c \mathcal{E}_{N}^{k}(M) \oplus \mathcal{H}^{k}(M) \oplus \mathcal{E}_{D}^{k}(M),
$$

where

$$
\begin{aligned}
c \mathcal{E}_{N}^{k}(M) & =\left\{\omega \in \Omega^{k}(M): \omega=\delta \xi \text { for some } \xi \in \Omega^{k+1}(M) \text { with } i^{*} \star \xi=0\right\} \\
\mathcal{H}^{k}(M) & =\left\{\omega \in \Omega^{k}(M): d \omega=0, \delta \omega=0\right\} \\
\mathcal{E}_{D}^{k}(M) & =\left\{\omega \in \Omega^{k}(M): \omega=d \zeta \text { for some } \zeta \in \Omega^{k-1}(M) \text { with } i^{*} \zeta=0\right\} .
\end{aligned}
$$

Equations (21) and (22) imply that

$$
0=i^{*} \delta \omega=i^{*} \delta(\delta \xi+\kappa+d \zeta)=i^{*} \delta d \zeta .
$$

Since $\delta d \zeta$ is co-exact and since the space of co-exact $k$-forms is precisely the orthogonal complement of the space of $k$-forms satisfying a Dirichlet boundary condition, (23) implies that $\delta d \zeta=0$. Hence, $d \zeta$ is co-closedbut $\mathcal{E}_{D}^{k}(M)$ is precisely the orthogonal complement of the space of co-closed $k$-forms, so it follows that $d \zeta=0$.

Therefore,

$$
\omega=\delta \xi+\kappa
$$

is co-closed. Since both $\omega$ and $\delta \xi \in c \mathcal{E}_{N}^{k}(M)$ satisfy a Neumann boundary condition, $\kappa$ must be a harmonic Neumann field. Moreover, since both $\omega$ and $\kappa$ are harmonic, it follows that $\delta \xi$ is harmonic. Hence,

$$
\omega=\delta \xi+\kappa \in\left(c \mathcal{E}_{N}^{k}(M) \cap \operatorname{ker} \Delta\right) \oplus \mathcal{H}_{N}^{k}(M)
$$

and so

$$
\varphi=i^{*} \omega \in i^{*}\left(c \mathcal{E}_{N}^{k}(M) \cap \operatorname{ker} \Delta\right)+i^{*} \mathcal{H}_{N}^{k}(M) .
$$

Conversely, forms in this space are clearly in the kernel of $\Psi$.

In (24) the sum of spaces is not, a priori, direct, but directness of the sum follows immediately from the fact that harmonic forms are uniquely determined by their boundary values [Sch95, Theorem 3.4.10].

The term $i^{*} \mathcal{H}_{N}^{k}(M)=\operatorname{ker} \Phi_{k}$ in (24) is exactly the space described in (i), so the lemma will follow from showing that $i^{*}\left(c \mathcal{E}_{N}^{k}(M) \cap \operatorname{ker} \Delta\right)$ is the direct sum of the spaces described in (ii) and (iii). 
Suppose, then, that $\varphi \in i^{*}\left(c \mathcal{E}_{N}^{k}(M) \cap \operatorname{ker} \Delta\right)$; i.e., that $\omega=\delta \xi$. Since $0=\Delta \omega=\Delta \delta \xi$, we know that

$$
0=(d \delta+\delta d) \delta \xi=\delta d \delta \xi,
$$

so $d \delta \xi$ is co-closed, meaning that $d \delta \xi \in \mathcal{H}^{k+1}(M)$; specifically, $d \delta \xi \in \mathcal{E H}^{k+1}(M)$. On the other hand, for any $d \gamma \in \mathcal{E} \mathcal{H}^{k+1}(M)$, there is a unique choice of primitive $\gamma$ that is in $c \mathcal{E}_{N}^{k}(M) \cap \operatorname{ker} \Delta$. Hence,

$$
c \mathcal{E}_{N}^{k}(M) \cap \operatorname{ker} \Delta \simeq \mathcal{E} \mathcal{H}^{k+1}(M) .
$$

In turn, since forms in $c \mathcal{E}_{N}^{k}(M) \cap \operatorname{ker} \Delta$ are uniquely determined by their pullbacks to the boundary, this implies that

$$
i^{*}\left(c \mathcal{E}_{N}^{k}(M) \cap \operatorname{ker} \Delta\right) \simeq \mathcal{E} \mathcal{H}^{k+1}(M) .
$$

Applying the Hodge star to the space $c \mathcal{E}_{N}^{k}(M) \cap \operatorname{ker} \Delta$ yields Cappell, DeTurck, Gluck, and Miller's space EHarm ${ }^{n-k}$. Thinking in those terms, $\delta \xi \in c \mathcal{E}_{N}^{k}(M)$ is a harmonic, co-exact form, but the primitive $\xi$ is not necessarily harmonic. There are two possibilities:

Case 1: If $\xi$ is harmonic, then

$$
0=\Delta \xi=(d \delta+\delta d) \xi=d \delta \xi+\delta d \xi,
$$

meaning that $d \delta \xi=-\delta d \xi$ is both exact and co-exact. Since $\Delta \delta \xi=$ 0 , this means that $\delta \xi$ has a conjugate form (in the sense of [BS08, Section 5]). This implies that $i^{*} \delta \xi \in \operatorname{ker} G_{k}$ [BS08, Theorem 5.1]. Since $\delta \xi$ is orthogonal to the space of closed $k$-forms on $M$, we have

$$
\varphi=i^{*} \delta \xi \in \operatorname{ker} G_{k} \cap i^{*}\left(\left(\mathcal{C}^{k}(M)\right)^{\perp}\right),
$$

which is the space in (ii).

Conversely, if $\varphi \in \operatorname{ker} G_{k} \cap i^{*}\left(\left(\mathcal{C}^{k}(M)\right)^{\perp}\right)$, then $\varphi=i^{*} \delta \xi$ for some $\delta \xi \in c \mathcal{E}_{N}^{k}(M)$ which has a conjugate form. This implies that $d \delta \xi$ is both exact and co-exact, and it is straightforward to check that $\xi$ can be chosen to be harmonic.

Case 2: If $\xi$ is not harmonic, then it belongs to the space

$$
\mathcal{N}^{k}:=\left\{\delta \xi \in c \mathcal{E}_{N}^{k}(M) \cap \operatorname{ker} \Delta: \Delta \xi \neq 0\right\} .
$$

This space is isomorphic to $H^{k+1}(M, \partial M ; \mathbb{R})$ [CDGM06, Lemma 3], and so $i^{*} \mathcal{N}^{k}$ is the space given in (iii).

The directness of the sum

$$
\left(\operatorname{ker} G_{k} \cap i^{*}\left(\left(\mathcal{C}^{k}(M)\right)^{\perp}\right)\right)+i^{*} \mathcal{N}^{k}
$$

again follows from the fact that harmonic forms are uniquely determined by their boundary values.

We can now determine the image of $\Psi_{k+1}$. 
Proof of Lemma 4.2. Suppose $\vartheta \in \Omega^{k}(\partial M)$ such that $\vartheta=\Psi \varphi$ for some $\varphi \in \Omega^{k+1}(\partial M)$. If $\omega \in \Omega^{k+1}(M)$ solves the boundary value problem (4), then $\vartheta=\Psi \varphi=i^{*} \delta \omega$.

Since $\omega$ satisfies a Neumann boundary condition,

$$
\delta \omega \in c \mathcal{E}_{N}^{k}(M)
$$

Moreover, since $\Delta$ commutes with the co-differential,

$$
\Delta \delta \omega=\delta \Delta \omega=0
$$

and so

$$
\delta \omega \in c \mathcal{E}_{N}^{k}(M) \cap \operatorname{ker} \Delta .
$$

Since $\omega$ is itself harmonic, this is precisely the situation described in Case 1 of the proof of Lemma 4.1, so

$$
\vartheta=i^{*} \delta \omega \in \operatorname{ker} G_{k} \cap i^{*}\left(\left(\mathcal{C}^{k}(M)\right)^{\perp}\right) .
$$

Conversely, if $\vartheta=i^{*} \delta \zeta$ for $\delta \zeta \in c \mathcal{E}_{N}^{k}(M) \cap \operatorname{ker} \Delta$ with $\zeta$ harmonic, then

$$
\Delta \zeta=0 \text { and } i^{*} \star \zeta=0,
$$

so $\vartheta=i^{*} \delta \zeta=\Psi i^{*} \zeta$ is in the image of $\Psi$.

\section{Corollary 4.3.}

$$
\operatorname{ker} \Phi_{k} \subset \operatorname{ker} \Psi_{k} \quad \text { and } \quad \operatorname{im} \Psi_{k} \subset \operatorname{im} \Phi_{n-k} .
$$

Proof. The fact that ker $\Phi_{k} \subset$ ker $\Psi_{k}$ is an immediate consequence of Lemma 4.1.

Now, suppose $\varphi \in \operatorname{im} \Psi_{k}$. Then, by Lemma $4.2, \varphi \in \operatorname{ker} G_{k-1}$, meaning $\varphi=i^{*} \omega$ for $\omega \in \Omega^{k-1}(M)$ satisfying

$$
\Delta \omega=0, \quad \delta \omega=0, \quad \text { and } \quad d \omega=\star d \eta
$$

for some $\eta \in \Omega^{n-k-1}(M)$ with $\Delta \eta=0$ and $\delta \eta=0$ [BS08, Theorem 5.1]. Therefore, for any $\lambda_{N} \in \mathcal{H}_{N}^{n-k}(M)$,

$$
\int_{\partial M} \varphi \wedge i^{*} \lambda_{N}= \pm \int_{\partial M} i^{*} \omega \wedge i^{*}\left(\star \star \lambda_{N}\right)= \pm\left[\left\langle d \omega, \star \lambda_{N}\right\rangle_{L^{2}(M)}-\left\langle\omega, \delta \star \lambda_{N}\right\rangle_{L^{2}(M)}\right]
$$

by Green's formula. The second term on the right hand side vanishes since $\lambda_{N}$ is closed, while the first is equal to

$$
\left\langle\star d \eta, \star \lambda_{N}\right\rangle_{L^{2}(M)}=\left\langle d \eta, \lambda_{N}\right\rangle_{L^{2}(M)}=0 .
$$

The first equality above is due to the fact that $\star$ is an isometry and the second follows because $\mathcal{H}_{N}^{n-k}(M)$ is orthogonal to the space of exact forms on $M$.

Putting (25) and (26) together shows that

$$
\int_{\partial M} \varphi \wedge i^{*} \lambda_{N}=0
$$

for any $\lambda_{N} \in \mathcal{H}_{N}^{n-k}(M)$, so Lemma 3.1 implies that $\varphi \in \operatorname{im} \Phi_{n-k}$, as desired. 


\section{Cochain MAPS AND The ADJOINT OF $\Psi$}

Since $\Psi$ is a chain map whose homologies are interesting, it seems natural to try to find associated cochain maps and compute their cohomologies. In fact, there are two such maps,

$$
\widetilde{\Psi}:=(-1)^{k(n-1)} \star_{\partial} \Psi \star_{\partial} \quad \text { and } \quad \Theta:=(-1)^{(k+1)(n-1)} \Phi \Psi \Phi .
$$

By definition both are maps $\Omega^{k}(\partial M) \rightarrow \Omega^{k+1}(\partial M)$.

5.1. The operator $\widetilde{\Psi}$. The fact that $\widetilde{\Psi}^{2}=0$ is immediate:

$$
\widetilde{\Psi}^{2}= \pm \star_{\partial} \Psi \star_{\partial} \star_{\partial} \Psi \star_{\partial}= \pm \star_{\partial} \Psi^{2} \star_{\partial}=0
$$

since $\Psi^{2}=0$.

Let $\widetilde{\Psi}^{k}$ be the restriction of $\widetilde{\Psi}$ to $\Omega^{k}(\partial M)$. Since $\star_{\partial}$ is an isomorphism,

$$
\operatorname{ker} \widetilde{\Psi}^{k} \simeq \operatorname{ker} \Psi_{n-k-1} \text { and } \operatorname{im} \widetilde{\Psi}^{k-1} \simeq \operatorname{im} \Psi_{n-k}
$$

and so

$$
H^{k}\left(\Omega^{*}(\partial M), \widetilde{\Psi}\right) \simeq H_{n-k-1}\left(\Omega^{*}(\partial M), \Psi\right) .
$$

Thus, we can use Theorem 2 to determine the cohomology groups of $\widetilde{\Psi}$.

Proposition 5.1. The cohomology groups of the cochain complex $\left(\Omega^{*}(\partial M), \widetilde{\Psi}\right)$ are

$$
H^{k}\left(\Omega^{*}(\partial M), \widetilde{\Psi}\right) \simeq H^{n-k}(M ; \mathbb{R}) \oplus H^{n-k-1}(M, \partial M ; \mathbb{R})
$$

The obvious guess, suggested by experience with $\Lambda$ and by the duality given in (27), is that $\widetilde{\Psi}$ is the adjoint of $\Psi$.

Proposition 5.2. $\widetilde{\Psi}$ is the adjoint of $\Psi$.

Proof. The proof follows along similar lines to the proof that $\Lambda^{*}=\star_{\partial} \Lambda \star_{\partial}$ [BS08, p. 132].

Let $\varphi \in \Omega^{k}(\partial M)$ and $\psi \in \Omega^{n-k}(\partial M)$. Suppose $\omega \in \Omega^{k}(M)$ solves the boundary value problem (4) and that $\eta \in \Omega^{n-k}(M)$ solves the equivalent boundary value problem for $\psi$.

The key step is to show that

$$
(-1)^{k+1} \int_{\partial M} \varphi \wedge \Psi \psi=(-1)^{k n+n+1} \int_{\partial M} \psi \wedge \Psi \varphi .
$$

Provided this is true, we can re-write the above equation as

$$
(-1)^{k n+k+1}\left\langle\varphi, \star_{\partial} \Psi \psi\right\rangle_{L^{2}(\partial M)}=-\left\langle\psi, \star_{\partial} \Psi \varphi\right\rangle_{L^{2}(\partial M)}
$$

or, equivalently,

$$
\left\langle\varphi, \star_{\partial} \Psi \psi\right\rangle_{L^{2}(\partial M)}=(-1)^{k(n-1)}\left\langle\psi, \star_{\partial} \Psi \varphi\right\rangle_{L^{2}(M)} .
$$

Letting $\psi=\star_{\partial} \psi^{\prime}$, this becomes

$\left\langle\psi, \star_{\partial} \Psi \star_{\partial} \psi^{\prime}\right\rangle_{L^{2}(\partial M)}=(-1)^{k(n-1)}\left\langle\star_{\partial} \psi^{\prime}, \star_{\partial} \Psi \varphi\right\rangle_{L^{2}(\partial M)}=(-1)^{k(n-1)}\left\langle\psi^{\prime}, \Psi \varphi\right\rangle_{L^{2}(\partial M)}$, 
since $\star_{\partial}$ is an isometry. Therefore,

$$
\Psi^{*}=(-1)^{k(n-1)} \star_{\partial} \Psi \star_{\partial}=\widetilde{\Psi},
$$

as desired.

To prove (28) we note that, by Green's formula,

$$
\begin{aligned}
\int_{\partial M} \varphi \wedge \Psi \psi=\int_{\partial M} i^{*} \omega \wedge i^{*} \delta \eta & =(-1)^{n(k+1)+n+1} \int_{\partial M} i^{*} \omega \wedge i^{*}(\star d \star \eta) \\
& =(-1)^{k n+1}\left(\langle d \omega, d \star \eta\rangle_{L^{2}(M)}-\langle\omega, \delta d \star \eta\rangle_{L^{2}(M)}\right) .
\end{aligned}
$$

Notice that

$$
-\langle\omega, \delta d \star \eta\rangle_{L^{2}(M)}=\langle\omega, d \delta \star \eta\rangle_{L^{2}(M)}
$$

since $0=\star \Delta \eta=\Delta \star \eta=d \delta \star \eta+\delta d \star \eta$. In turn,

$$
\langle\delta \omega, \delta \star \eta\rangle_{L^{2}(M)}=\langle\omega, d \delta \star \eta\rangle_{L^{2}(M)}-\int_{\partial M} i^{*} \delta \star \eta \wedge i^{*} \star \omega .
$$

Since $i^{*} \star \omega=0$, the second term on the right hand side vanishes. Therefore, we can re-write (29) as

$$
\int_{\partial M} \varphi \wedge \Psi \psi=(-1)^{k n+1}\left(\langle d \omega, d \star \eta\rangle_{L^{2}(M)}+\langle\delta \omega, \delta \star \eta\rangle_{L^{2}(M)}\right) .
$$

Completely analogous reasoning yields the expression

$$
\int_{\partial M} \psi \wedge \Psi \varphi=(-1)^{k n+n+1}\left(\langle d \eta, d \star \omega\rangle_{L^{2}(M)}+\langle\delta \eta, \delta \star \omega\rangle_{L^{2}(M)}\right)
$$

Therefore, (28) follows from (30) and (31) because

$$
\begin{aligned}
& \langle d \omega, d \star \eta\rangle_{L^{2}(M)}=\left\langle\star d \omega, \star d \star \eta\left\langle_{L^{2}(M)}=(-1)^{k(n+1)}\langle\delta \star \omega, \delta \eta\rangle_{L^{2}(M)}\right.\right. \\
& \langle\delta \omega, \delta \star \eta\rangle_{L^{2}(M)}=\langle\star \delta \omega, \star \delta \star \eta\rangle_{L^{2}(M)}=(-1)^{k(n+1)}\langle d \star \omega, d \eta\rangle_{L^{2}(M)}
\end{aligned}
$$

(the first equality in each line is due to the fact that $\star$ is an isometry).

5.2. The operator $\Theta$. The are several different equivalent ways of expressing the operator $\Theta=(-1)^{(k+1)(n+1)} \Phi \Psi \Phi$. Using (9),

$$
\Theta=(-1)^{(k+1)(n+1)} \Phi \Psi \Phi=(-1)^{k n} d_{\partial} \Phi^{2} .
$$

On the other hand, using (11),

$$
\Theta=(-1)^{(k+1)(n+1)} \Phi \Psi \Phi=(-1)^{n(k+1)} \Phi^{2} d_{\partial} .
$$

Finally, combining (12) with (33) yields

$$
\Theta=(-1)^{n(k+1)} \Phi^{2} d_{\partial}=\left(d_{\partial} \Psi+\Psi d_{\partial}\right) d_{\partial}=d_{\partial} \Psi d_{\partial} .
$$

This last expression makes it clear that $\Theta$ is a cochain map:

$$
\Theta^{2}=d_{\partial} \Psi d_{\partial} d_{\partial} \Psi d_{\partial}=0 .
$$

Proposition 5.3. The cohomology of the cochain complex $\left(\Omega^{*}(\partial M), \Theta\right)$ is given, up to isomorphism, by

$$
H^{k}\left(\Omega^{*}(\partial M), \Theta\right) \simeq H^{k+1}(M, \partial M ; \mathbb{R}) \oplus H^{k}(M ; \mathbb{R}) .
$$


Notice that $\left(\Omega^{*}(\partial M), \Theta\right)$ has the same cohomology as $\left(\Omega^{*}(\partial M), \widetilde{\Psi}\right)$.

We omit the proof of Proposition 5.3, which is somewhat long and technical, though not particularly difficult. Two perhaps surprising consequences are:

(i) Since $\Theta$ has the same cohomology as $\widetilde{\Psi}$, the homology of $\Psi$ can be completely recovered from that of $\Theta$. However, by (34), $\Theta=$ $d_{\partial} \Psi d_{\partial}$, so pre- and post-composing $\Psi$ by $d_{\partial}$ does not change the (co)homology.

(ii) By (32) and (33),

$$
\Theta= \pm d_{\partial} \Phi^{2}= \pm \Phi^{2} d_{\partial}
$$

Hence, the homology of $\Psi$ is completely determined by the operator $\Phi$, and the results of Corollaries 3 and 4 depend only on $\Phi$. In that spirit, the following is a restatement of the $k=0$ case of Corollary 3 .

Corollary 5. A copy of the cohomology group $H^{n-1}(M ; \mathbb{R})$ is distinguished by the operator $\Phi$ inside $\Omega^{0}(\partial M)$, the space of smooth functions on $\partial M$. Specifically,

$$
\operatorname{ker}\left(d_{\partial} \Phi^{2}\right) / \operatorname{ker} \Phi \simeq H^{n-1}(M ; \mathbb{R})
$$

\section{REFERENCES}

[Bel03] Mikhail Belishev, The Calderon problem for two-dimensional manifolds by the BC-method, SIAM J. Math. Anal. 35 (2003), no. 1, 172-182, doi:10.1137/S0036141002413919.

[BS08] Mikhail Belishev and Vladimir Sharafutdinov, Dirichlet to Neumann operator on differential forms, Bull. Sci. Math. 132 (2008), no. 2, 128-145, doi:10.1016/j.bulsci.2006.11.003.

[Cal80] Alberto P. Calderón, On an inverse boundary value problem, Seminar on Numerical Analysis and its Applications to Continuum Physics, Soc. Brasileira de Matemática, Rio de Janeiro, 1980, pp. 65-73. Republished in Comput. Appl. Math. 25 (2006), no. 2-3, 133-138, doi:10.1590/S0101-82052006000200002.

[CDGM06] Sylvain Cappell, Dennis DeTurck, Herman Gluck, and Edward Y. Miller, Cohomology of harmonic forms on Riemannian manifolds with boundary, Forum Math. 18 (2006), no. 6, 923-931, doi:10.1515/FORUM.2006.046.

[JL05] Mark S. Joshi and William R.B. Lionheart, An inverse boundary value problem for harmonic differential forms, Asymptot. Anal. 41 (2005), no. 2, 93-106.

[KLU10] Katsiaryna Krupchyk, Matti Lassas, and Gunther Uhlmann, Inverse problems for differential forms on Riemannian manifolds with boundary, Preprint, arXiv:1007.0979 [math.AP], 2010.

[LTU03] Matti Lassas, Michael Taylor, and Gunther Uhlmann, The Dirichlet-toNeumann map for complete Riemannian manifolds with boundary, Comm. Anal. Geom. 11 (2003), no. 2, 207-221.

[LU01] Matti Lassas and Gunther Uhlmann, On determining a Riemannian manifold from the Dirichlet-to-Neumann map, Ann. Sci. École Norm. Sup. (4) 34 (2001), no. 5, 771-787, doi:10.1016/S0012-9593(01)01076-X.

[Sch95] Günter Schwarz, Hodge Decomposition-A Method for Solving Boundary Value Problems, Lecture Notes in Mathematics, vol. 1607, Springer-Verlag, Berlin, 1995. 
[Sha09] Vladimir Sharafutdinov, Linearized inverse problem for the Dirichlet-toNeumann operator on differential forms, Bull. Sci. math. 133 (2009), 419-444, doi:10.1016/j.bulsci.2008.07.001.

[Sho09] Clayton Shonkwiler, Poincaré duality angles for Riemannian manifolds with boundary, Preprint, arXiv:0909.1967 [math.DG], 2009.

Sobolev Institute of Mathematics

E-mail address: sharaf@math.nsc.ru

$U R L:$ http://www.math.nsc.ru/ ${ }^{\text {sharafutdinov/ }}$

Department of Mathematics, Haverford College

E-mail address: cshonkwi@haverford.edu

$U R L:$ http: //www.haverford.edu/math/cshonkwi/ 